\title{
Effects of zonation on the results of the application of the regional time predictable seismicity model in Greece and Japan
}

\author{
G. F. Karakaisis \\ Geophysical Laboratory, University of Thessaloniki, Thessaloniki 54006, Greece
}

(Received July 29, 1999; Revised November 15, 1999; Accepted January 21, 2000)

Recent work on time dependent seismicity is mainly based on the so-called "regional time predictable model", which is expressed by the relation:

$$
\log T_{t}=c M_{p}+a
$$

where $T_{t}$ is the interevent time, i.e. the time between two successive mainshocks of a seismogenic region, and $M_{p}$ is the magnitude of the precedent mainshock. Parameter $a$ is a function of the magnitude of the minimum earthquake considered and of the tectonic loading and $c$ is a positive $(\approx 0.3)$ constant. A problem of the method, as it has been applied till now, is its dependence on the zonation, that is, on the definition of the seismogenic regions, which is subjective to some extent. In the present work a different approach, which assumes no a-priori regionalization of the area, is attempted in order to check the validity of the model. Grids of equally spaced points at $0.5^{\circ}$ have been created for Greece and Japan and the mainshocks located within each circle with center at a point and radius varying between 30 and $150 \mathrm{~km}$ were considered. When the number of mainshocks within the circle was four or larger, regression was performed and the $c$ value was calculated. In about $75 \%$ of the cases for Greece with sufficient data and $80 \%$ for Japan the parameter $c$ was found to be positive. This result strongly supports the validity of the model.

\section{Introduction}

Papazachos (1989) proposed a regional time-dependent seismicity model to account for the temporal characteristics of the generation of strong earthquakes in seismogenic regions of the Aegean area. According to this model, the time of occurrence of the next mainshock in a certain seismogenic region is positively dependent on the magnitude of the last mainshock in this source. Since then the model has been developed, resulting in the "regional time- and magnitude predictable seismicity model", which has been tested and applied in almost all areas of the continental fracture system (see Papazachos and Papaioannou, 1993; Papazachos et al., 1997a, b and references therein). The essence of the model is that the interevent time $T_{t}$, and the magnitude, $M_{f}$ of the next mainshock in a seismogenic region depend on the magnitude, $M_{\min }$, of the minimum mainshock considered, the magnitude, $M_{p}$, of the last mainshock and the yearly seismic moment rate, $m_{o}$, in the region. It has to be noted that this model differs from the originally proposed time predictable model (Shimazaki and Nakata, 1980), in that it holds not only for an area containing a large fault where the characteristic earthquake occurs but for an area which includes, in addition to the large fault, smaller faults where smaller earthquakes occur. The model is expressed by the relations (1) and (2):

Copy right $\odot$ The Society of Geomagnetism and Earth, Planetary and Space Sciences (SGEPSS); The Seismological Society of Japan; The Volcanological Society of Japan; The Geodetic Society of Japan; The Japanese Society for Planetary Sciences.

$$
\begin{aligned}
& \log T_{t}=0.19 M_{\min }+0.33 M_{p}-0.39 \log m_{o}+q \\
& M_{f}=0.73 M_{\min }-0.28 M_{p}+0.40 \log m_{o}+m
\end{aligned}
$$

where the constants have been determined on the basis of a large data set consisting of 1811 observations $\left(T, M_{\min }\right.$, $M_{p}, M_{f}$ ) in 274 seismogenic regions (Papazachos et al., 1997a).The positive dependence of the interevent time, $T_{t}$, on the magnitude, $M_{p}$, of the preceding mainshock is denoted by the positive value of the parameter $c$. The global applicability of the model has been successfully tested (Papazachos and Papadimitriou, 1997).

A problem related to this model is that the procedure of regionalization, that is, the definition of the seismogenic regions, is subjective to some extent despite the fact that it is based on combined seismological and tectonic information of the area studied. To check the effect of regionalization on the main features of the model, different divisions were made in areas with complex seismotectonic regime. It was observed that although the accurate definition of seismogenic regions is important, it is not very critical for the main characteristics of the model or for the results obtained from its application (Papazachos et al., 1997a).

The present paper aims at further clarifying this matter, following an approach which assumes no a-priori regionalization of the studied area, that is, without taking into account any information concerning the area, even in the case where clear seismotectonic features suggest that 
certain parts of this area, such as regions where well defined active faults exist, merit particular treatment.

The areas of Greece and Japan have been selected to test this approach, since zonation for these areas is based on abundant seismotectonic and topographic-bathymetric information enabling a sufficiently accurate definition of seismogenic regions.

\section{Greece-Data and Method}

In the data bank of the Geophysical Laboratory of the Aristotle University of Thessaloniki there is information on the basic focal parameters (origin time, epicenter, focal depth, magnitude) of more than 40,000 earthquakes which occurred in the broader Aegean area during 550BC-1995 with magnitudes in the range 3.0-8.3. Not all the data, however, have the basic properties (completeness, homogeneity, accuracy) which are required for reliable seismicity studies in the broader Aegean area (Papazachos and Papazachou, 1997). For this reason, an updated catalogue has very recently been compiled which has such properties. From this catalogue, only shallow earthquakes ( $h<60 \mathrm{~km}$ ) with $M \geq 5.5$ which occurred in Greece and its surroundings have been selected for the present study. The resulting data set is complete for the following time intervals and cutoff magnitudes:
$1911-1995, M \geq 5.5$,

$1845-1995, M \geq 6.5$.

The epicenters of these earthquakes are shown in Fig. 1. On the same map, the 67 seismogenic regions in which Aegean and surrounding areas have been divided, were also drawn. These regions have recently been defined by Papazachos and Papaioannou (1997) on the basis of new seismological data as well as on additional geological and geomorphological information.

On this map a grid of equally spaced points (denoted by crosses) at $0.5^{\circ}$ was created. Each point was taken as the center of a circle with radius increasing from 30 to $100 \mathrm{~km}$ in steps of $10 \mathrm{~km}$, and all earthquakes within each circle were considered. The next stage was to decluster the data, that is, to define the mainshocks, preshocks (foreshocks in the broad sense) and postshocks (aftershocks in the broad sense). As it is known, clustering is usually attributed to foreshocks and mainly to aftershocks in the strict sense, that is, to earthquakes that have their foci in the rupture zone of the mainshock and usually last a few months. However, even when these shocks are omitted from the data, clustering still prevails (Kagan and Jackson, 1991), because spatial clustering occurs not only in the rupture zone of a mainshock but also in a broader area (seismogenic region) where other mainshocks also occur, resulting in a

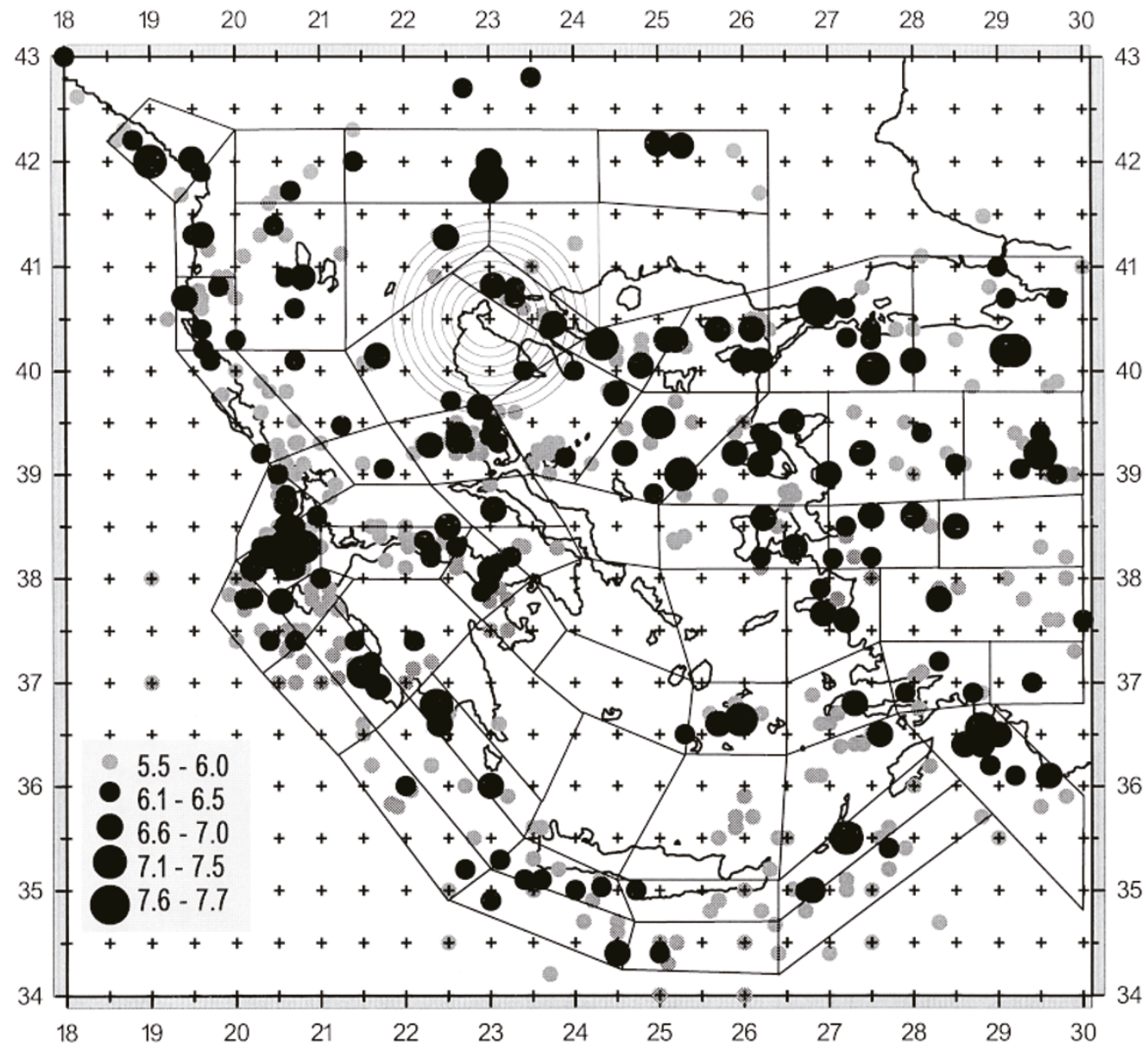

Fig. 1. The epicenters of strong shallow earthquakes which occurred after 1911 with $M \geq 5.5$ and after 1845 with $M \geq 6.5$ in the area studied, along with the 67 seismogenic regions which have recently been defined by Papazachos and Papaioannou (1997). The crosses denote the centers of the circles with radii from 30 to $100 \mathrm{~km}$. Circles with radii $30-100 \mathrm{~km}$, with a step of $10 \mathrm{~km}$, have been drawn with their center located at $40.5^{\circ} \mathrm{N}-$ $23.0^{\circ} \mathrm{E}$. 
time clustering of the order of several years. In the present study, declustering of the data for each circle is based on the relations (Karakaisis et al., 1991; Papazachos et al., 1997a):

$$
t_{p}=3 \text { years, } \log t_{a}=0.06+0.13 M_{p}
$$

where $t_{p}$ and $t_{a}$ are the durations of the preshock and the postshock activity, respectively.

A constant duration of the preshock activity and an increase of the duration of the postshock activity with the magnitude of the preceding mainshock is expected by the time predictable model (Mogi, 1985). According to relations (3) the total duartion of this clustering may reach up to 20 years. In practice, however, it is mostly of the order of 15 years.

The declustering procedure is performed in the following way: the largest event in the catalogue for a certain seismogenic region is first picked up and its preshocks and postshocks are determined by using the relations (3). For instance, if the largest event is an earthquake with $M=7.0$, then all events which occurred 3 years before and
9.3 years after this earthquake are considered as its preshocks and postshocks. Thus, one of the seismic sequences of this region is defined and a representative magnitude, $M$, for this sequence is determined by summing all seismic moments of the shocks of the sequence, as they have been calculated by a method proposed by Molnar (1979). This magnitude, $M$, is considered as the magnitude of the mainshock $\left(M_{p}\right)$. Then, the second largest earthquake of the remaining part of the catalogue is picked up and the procedure is continued till every event of the catalogue has become a member of a group which includes a mainshock and its preshocks and postshocks.

For each circle the earthquakes were first considered and then, after declustering, the mainshocks were found. The smallest mainshock of the $n$ mainshocks is taken as $M_{\min 1}$ and the interevent times, $T$, between successive mainshocks with magnitudes equal to or larger than $M_{\min 1}$ are calculated. Then, the second smallest mainshock is considered as $M_{\min 2}$ with $M_{\min 2}>M_{\min 1}$ and new interevent times between successive mainshocks with magnitudes equal to or larger than $M_{\min 2}$ are calculated. This procedure is continued until the last $M_{k}<M_{\max }$, with $k=n-1$, is

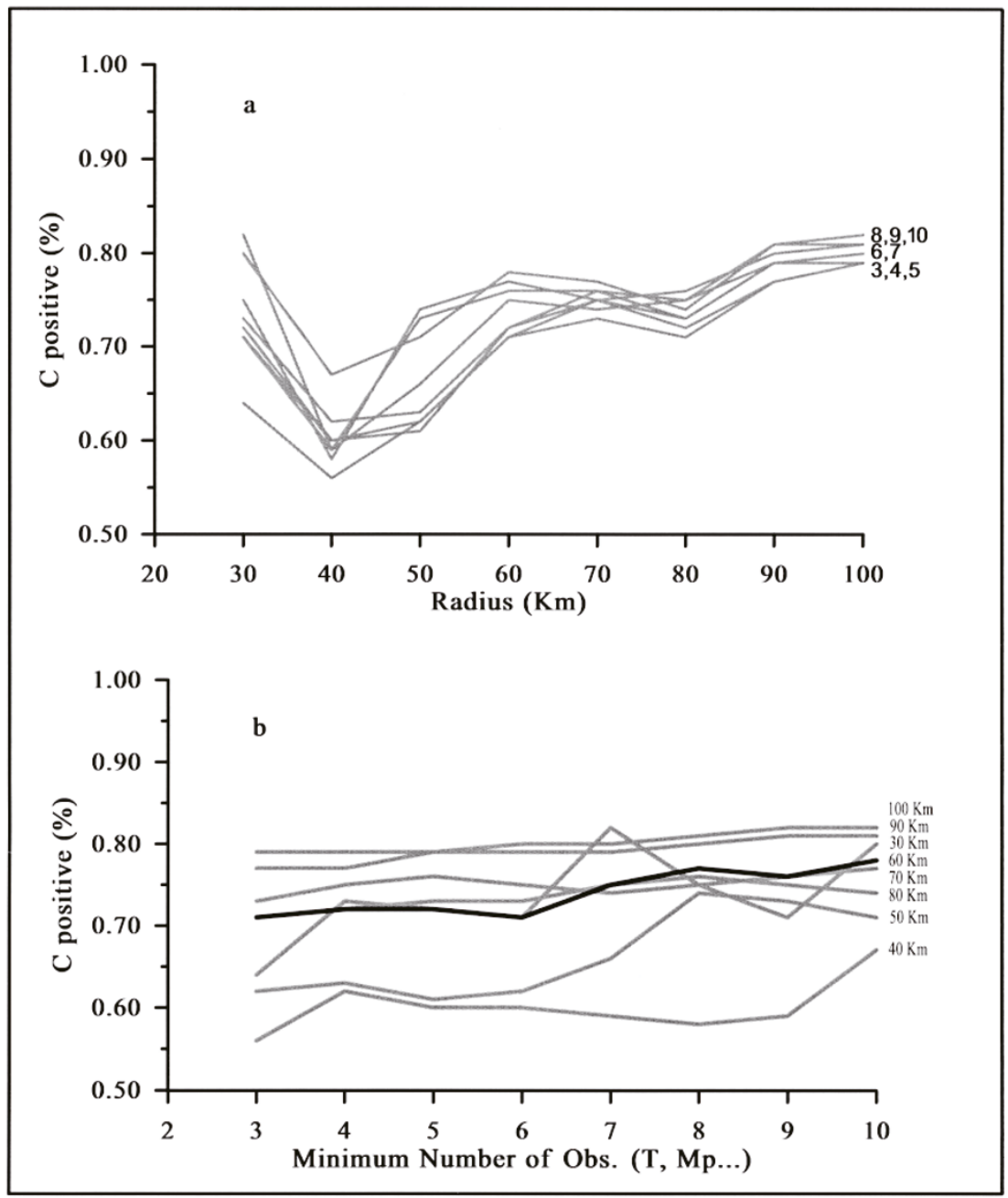

Fig. 2. (a) Plot of the ratio of the positive $c$-values to the sum of all $c$-values (positive+negative values) versus the radius of the circles considered for the Aegean area. The lines correspond to different minimum number of observations $\left(T, M_{\min }, M_{p}\right)$ that have been used in multilinear regression. (b) The same ratio (positive $c$-values to the sum of all $c$-values) plotted versus the minimum number of observations $\left(T, M_{\text {min }}, M_{p}\right.$ ) with the lines corresponding to different circle radii. 
considered as a $M_{\min k}$ for the respective circle. Thus, for each circle, a number of observations $\left(T, M_{\min }, M_{p}\right)$ resulted and, provided there were 4 or more, they were used as input in a multilinear regression routine compiled by $C$. Papazachos (personal communication), while the output of this routine was a respective number of $c$-values.

Figure $2 \mathrm{a}$ is a plot of the ratio of the positive $c$-values to the sum of all $c$-values (positive + negative values) versus the radius of the circles considered. The lines correspond to different minimum numbers of observations $\left(T, M_{\text {min }}\right.$, $M_{p}$ ) that have been used in the multilinear regression. It is observed that in the majority of the cases in all circles this ratio is larger than $55 \%$. It is also observed that for the circles with radii equal to or larger than $60 \mathrm{~km}$ more than $70 \%$ of the $c$-values are positive. The same ratio (positive $c$-values to the sum of all $c$-values) has been plotted versus the minimum number of observations $\left(T, M_{\min }, M_{p}\right)$ with the lines corresponding to different circle radii and is shown in Fig. 2b. It is observed that in the majority of the cases the positive $c$-values outnumber the negative ones. One may argue that these results may be biased since for larger $M_{\min }$ longer interervent times are expected. However, tests of this model on random catalogues underway, which will appear soon, show that its main feature (positive dependence of the return period on $M_{p}$ ) is not artificially derived.

\section{Japan-Data}

In a recent paper (Papazachos et al., 1994), the area of Japan had been divided into 12 seismogenic regions on the basis of geomorphological and seismotectonic information, of spatial clustering of epicenters of strong earthquakes, of the maximum earthquake ever observed and of the seismicity level. The major factors, however, which contributed to that zonation were the distribution of aftershock volumes and source areas of recent events, of tsunamigenic sources and of several, well documented, focal areas of historical earthquakes. For example, the seismogenic region 3 (shown in Fig. 3) was defined on the basis of the rupture zone of the 1707 Hoei earthquake described by Ando (1975). For a detailed description of the zonation see Papazachos et al. (1994) and references

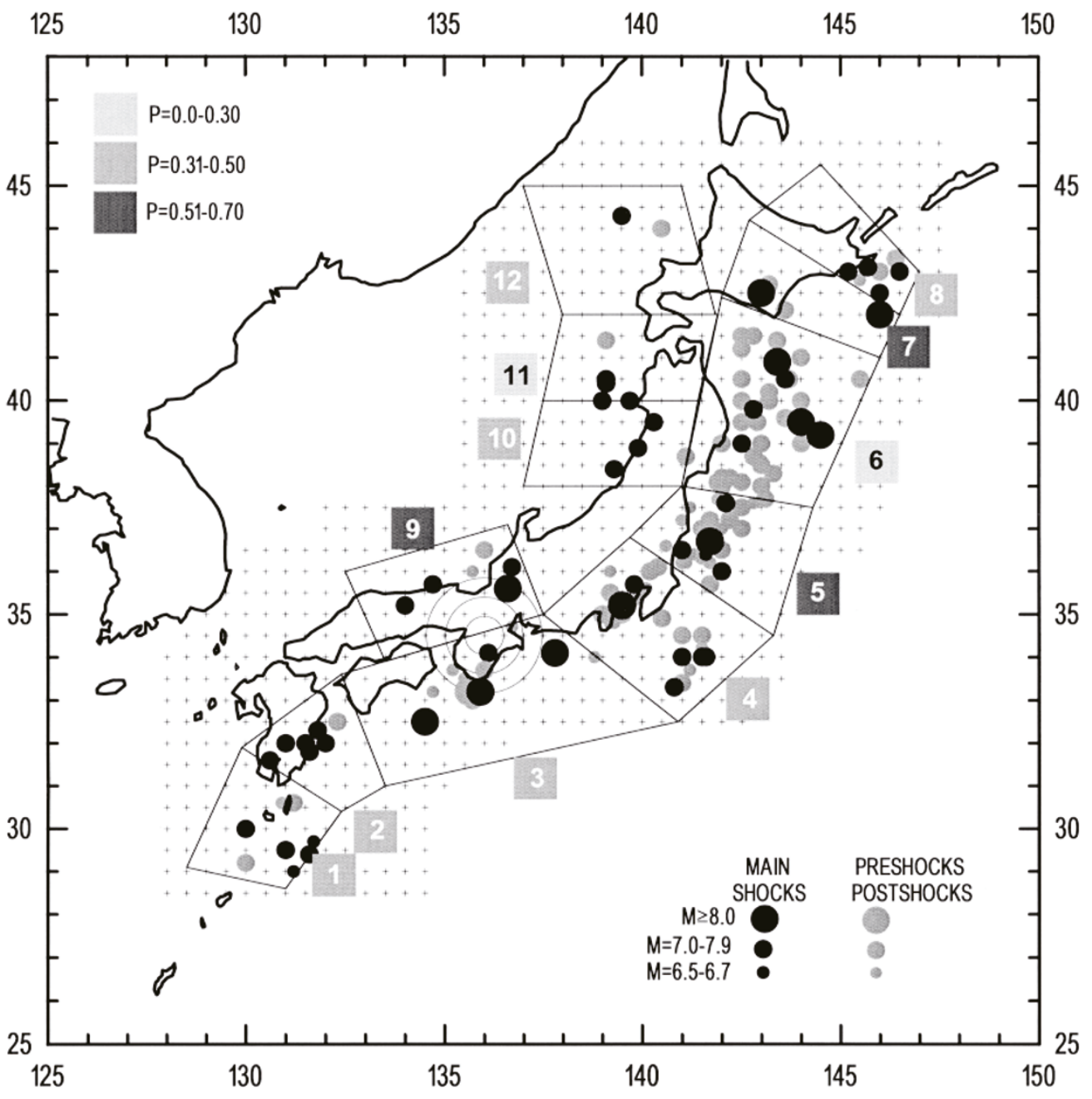

Fig. 3. Epicenters of strong shallow earthquakes which occurred in Japan and its surrounding area and have been considered in the present study. The black circles correspond to the mainshocks while the gray circles denote preshocks and postshocks in the sense described in the text. The 12 seismogenic regions defined by Papazachos et al. (1994) are also shown, along with the probabilities determined in their paper for the generation of strong $(M \geq 7.5)$ earthquakes during 1993-2002 (rectangles close to each seismogenic region). Circles with radii equal to 50, 100 and $150 \mathrm{~km}$ have been drawn with their center located at $34^{\circ} \mathrm{N}-136^{\circ} \mathrm{E}$. 
therein.

The data used in the present work have been taken from the catalogue of Pacheco and Sykes (1992), which lists all strong $(M \geq 7.0)$ events that occurred during 1900-1989. Magnitudes of historical earthquakes were taken from Rikitake (1976, 1982), while for the time period 18851900 the catalogue of Utsu (1988) was used. For events not listed in these catalogues, as well as for earthquakes with $M<7.0$, the catalogues of Abe (1981) and Tsapanos et al. (1990) were considered. Data of strong events during 1990-1992 were taken from the ISC bulletins. Examination of the completeness of all data collected revealed that the catalogue which finally is used in this work can be considered as complete for the following time intervals and cutoff magnitudes:

$$
\begin{aligned}
& 1951-1992, M \geq 6.0, \\
& 1931-1992, M \geq 6.5, \\
& 1885-1992, M \geq 7.0, \\
& 1840-1992, M \geq 7.8, \\
& 1700-1992, M \geq 8.5 .
\end{aligned}
$$

Figure 3 shows the epicenters of all strong earthquakes which were used in this study. Three magnitude ranges are depicted, while mainshocks and preshocks-postshocks, as they have previously been defined, are denoted by black and gray symbols, respectively. The 12 seismogenic regions, defined by Papazachos et al. (1994), are also shown. On this map, a grid of equally spaced points (denoted by crosses) at $0.5^{\circ}$ apart was created. Again, each point was taken as the center of circle with radius increasing from 30 to $150 \mathrm{~km}$ in steps of $10 \mathrm{~km}$, and all earthquakes which were inside each circle and fulfilled the aforementioned completeness criteria were considered. Then, following the declustering procedure described previously, the mainshocks in every circle were defined and the interevent times between successive mainshocks were determined, while regression was performed on these data and $c$-values were obtained.

The results (Figs. $4 \mathrm{a}$ and b) showed that, as in the case of Greece, the ratio of the positive $c$-values to the sum of all $c$-values (positive and negative $c$-values) is larger than $60 \%$ for all the circles considered, while this ratio was larger than $87 \%$ for circles with radius, $r \geq 100 \mathrm{~km}$.

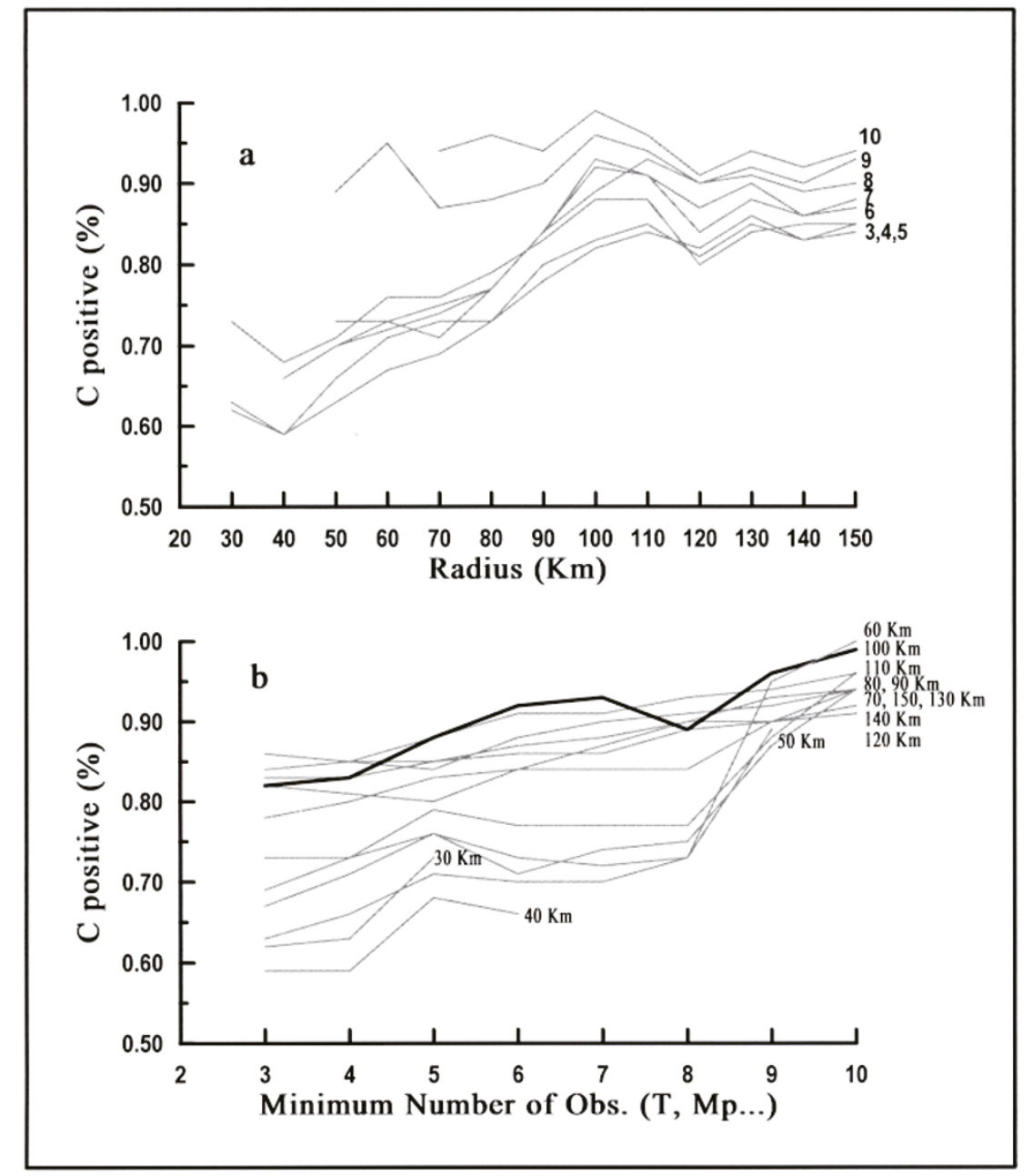

Fig. 4. (a) Plot of the ratio of the positive $c$-values to the sum of all $c$-values (positive+negative values) versus the radius of the circles considered for the area of Japan. The lines correspond to different minimum number of observations $\left(T, M_{\min }, M_{p}\right)$ that have been used in multilinear regression. (b) The same ratio (positive $c$-values to the sum of all $c$-values) plotted versus the minimum number of observations $\left(T, M_{\text {min }}, M_{p}\right.$ ) with the lines corresponding to different circle radii. 


\section{Calculation of Probabilities}

To further check the applicability of the model without taking into account any information concerning the regionalization of the area, probabilities for the generation of strong $(M \geq 6.0)$ shallow earthquakes in the broader Aegean area during the period 1996-2010 are calculated and the results are compared with probabilities determined for the seismogenic regions of this area, as they are drawn in Fig. 1.

Very recently, Papazachos and his colleagues (1997c) estimated probabilities for the generation of strong shallow and intermediate depth earthquakes in the broader Aegean area during the time period 1996-2010. Figure 5a shows, by rectangles of three shades located at the centers of the seismogenic regions of Fig. 1, the probability values for the occurrence of strong $(M \geq 6.0)$ shallow earthquakes during this time period. For the same period, 1996-2010, probabilities have been determined, for each circle with radius equal to $60 \mathrm{~km}$, since this was the smallest radius for which the results (Fig. 2a) were satisfactory. The procedure for the calculation of the probabilities is explained below.

It has been found (Papazachos and Papaioannou, 1993) that the ratio of the observed interevent time, $T$, to the theoretically calculated one, $T$, follows a lognormal distribution with a standard deviation, $\sigma$, equal to 0.29 . To calculate the conditional probability, $P$, that the repeat time $T$ of an earthquake will occur in a region during the next $\Delta t$ years, conditional on the $t$ years that have elapsed since the last earthquake, the following formula is used:

$$
P=\frac{F\left(L_{2} / \sigma\right)-F\left(L_{1} / \sigma\right)}{1-F\left(L_{1} / \sigma\right)}
$$

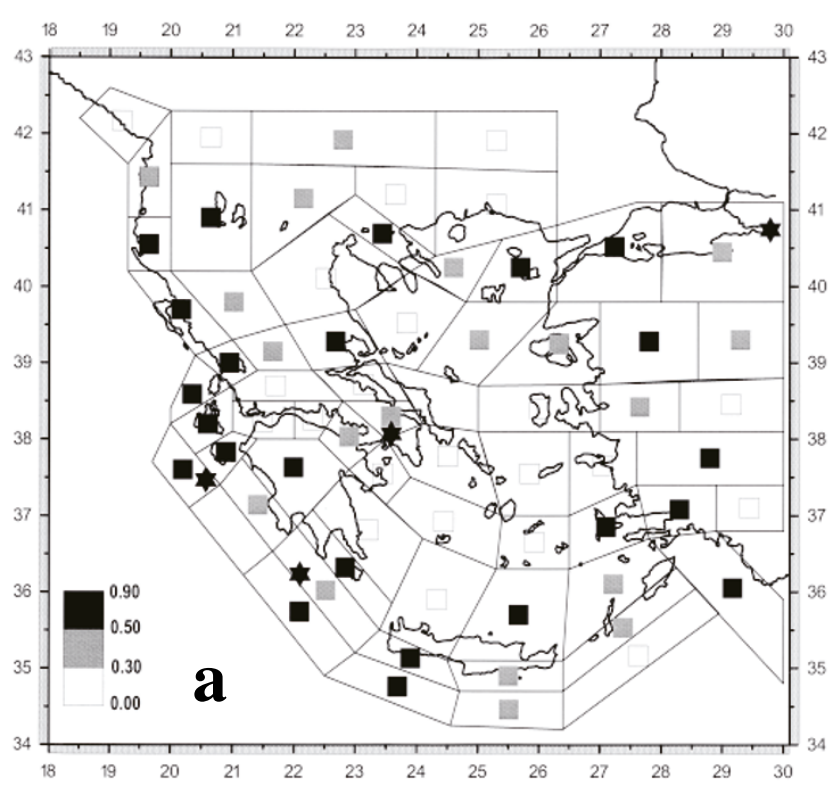

where $L_{2}=\log \left(t+\Delta t / T_{t}\right), L_{1}=\log \left(t / T_{t}\right)$, and $F$ is the complementary cumulative value. The probabilities thus calculated, which are denoted by rectangles of three shades located at the centers of the circles of Fig. 1, are shown in Fig. 5b. The similarity of the spatial distribution of probability values in both maps of Figs. 5a and b and especially for values equal to or larger than $50 \%$ is obvious. It has to be noted that four strong earthquakes occurred in regions where moderate or high probabilities had been estimated. These earthquakes, which are denoted by stars in Figs. 5a and b are: 1) 13 October 1997, $M_{s}=6.7$, $\left.36.38^{\circ} \mathrm{N}-22.07^{\circ} \mathrm{E}, 2\right) 18$ November $1997, M_{w}=6.7$, $\left.37.57^{\circ} \mathrm{N}-20.66^{\circ} \mathrm{E}, 3\right) 17$ August $1999, M_{w}=7.4,40.76^{\circ} \mathrm{N}-$ $\left.29.97^{\circ} \mathrm{E}, 4\right) 7$ September $1999, M_{s}=5.9,38^{w} .05^{\circ} \mathrm{N}-23.56^{\circ} \mathrm{E}$.

The same procedure was applied to the area of Japan. It was found that the ratio, $\mathrm{T} / T_{t}$, for all data obtained from the $100 \mathrm{~km}$-radius circles, i.e. the smallest radius for which the results from the regression are satisfactory (Fig. 4a), follows a lognormal distribution with a mean value equal to 0.0 and standard deviation equal to 0.27 . Probabilities for the generation of large $(M \geq 7.5)$ shallow earthquakes were calculated for each and every circle with radius equal to $100 \mathrm{~km}$, for the time period 19932002. This time period and cutoff magnitude were chosen in order to facilitate comparison with the probabilities calculated by Papazachos et al. (1994). The results of this calculation are shown in Fig. 6, where three different shade rectangles have been used to denote three different probability ranges (light gray: 0.0-30\%; gray: 30-50\%; dark gray: $50-80 \%$ ). The comparison of the distribution of the probability values calculated in the present work with the probabilities determined by Papazachos et al. (1994), which are denoted by the rectangles close to each

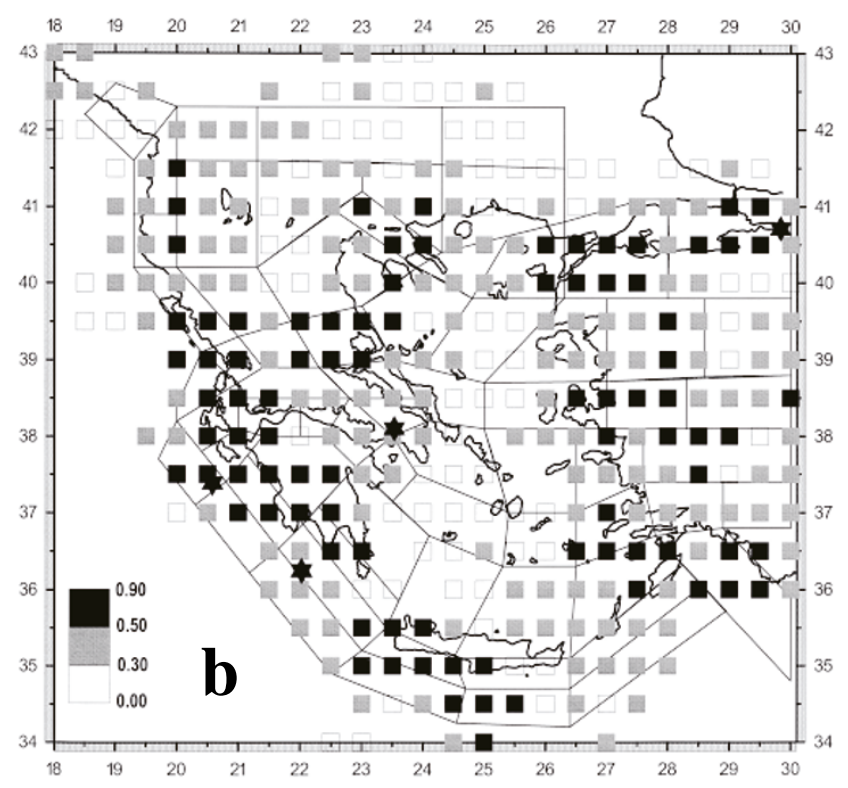

Fig. 5. (a) Probabilities for the generation of strong $(M \geq 6.0)$ shallow earthquakes in the 67 seismogenic regions of the Aegean area during 19962010 (Papazachos et al., 1997c). (b) Probabilities for the generation of strong $(M \geq 6.0)$ shallow earthquakes in circles having their centers at $0.5^{\circ}$ apart with radius equal to $60 \mathrm{~km}$ during 1996-2010. The stars in both figures show the epicenters of four strong earthquakes which occurred in the Aegean area since 1996. 


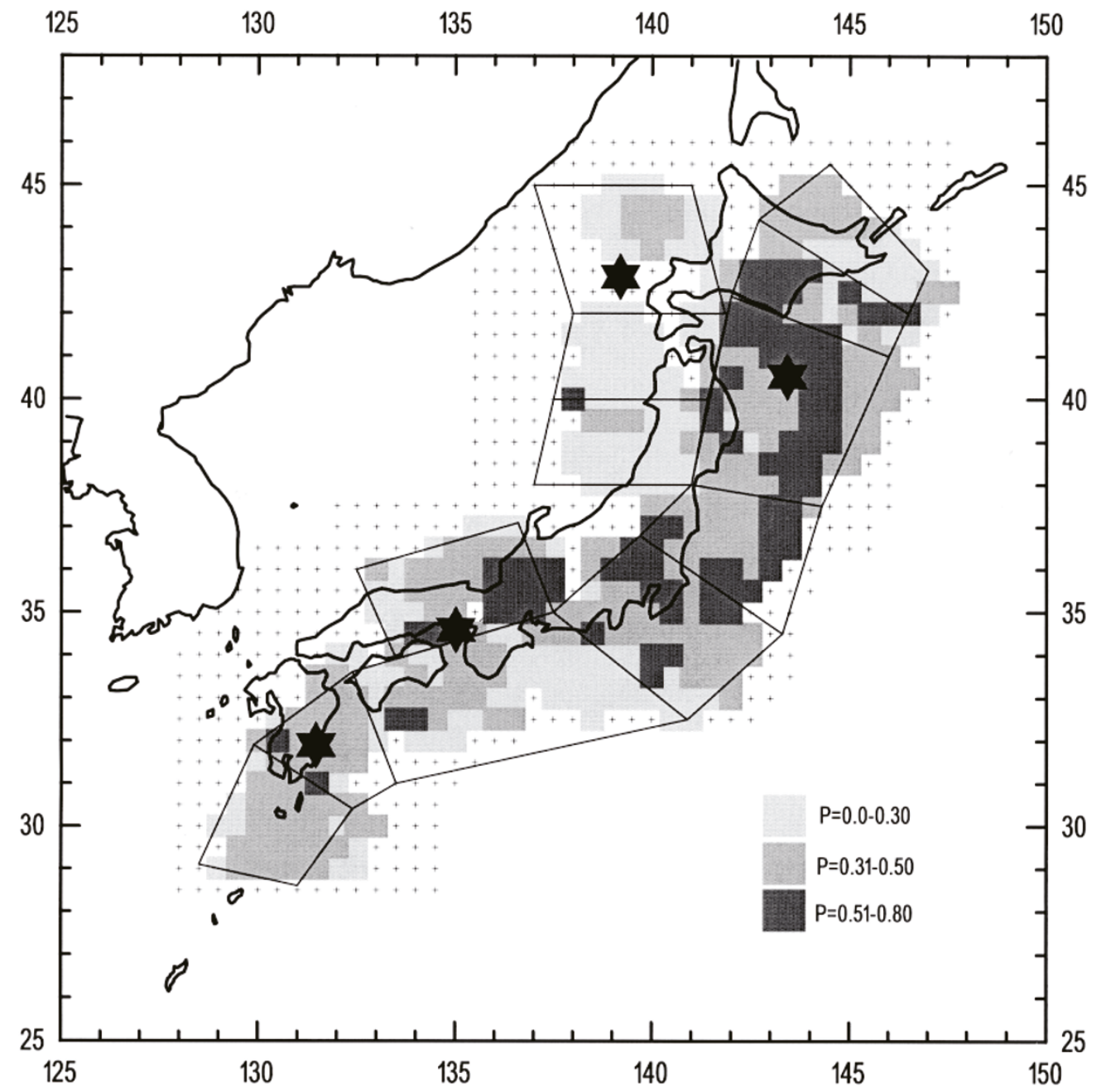

Fig. 6. Probabilities for the generation of strong $(M \geq 7.5)$ shallow earthquakes during 1993-2002, computed for circles having their centers at $0.5^{\circ}$ apart with radius equal to $100 \mathrm{~km}$. The stars denote the mainshock epicenters $(M \geq 7.0)$ which occurred in this area during 1993-1998.

seismogenic region in Fig. 3, seems satisfactory. However, differences are observed between the probability calculated by Papazachos et al. (1994) for the region 6 (Tohoku, in their paper) and the probabilities found in this work for the circles which are located within this region. This discrepancy is probably due to the fact that, in that paper, a $M=7.4$ event that occurred on 1 November 1989 had been taken as the last mainshock, thus resulting in a low $(P=22 \%)$ probability for the whole seismogenic region during 1993-2002, whereas in the present work the probabilities are low only in the circles that this event belongs to. It is again noted that, since 1993 the following large $(M \geq 7.0)$ mainshocks occurred inside the seismogenic regions defined by Papazachos et al. (1994): 1) 12 July $1993,42.85^{\circ} \mathrm{N}-139.20^{\circ} \mathrm{E}, M=7.7$; 2) 28 December 1994 , $40.53^{\circ} \mathrm{N}-143.42^{\circ} \mathrm{E}, M=7.8$; 3$) 16$ January $1995,34.58^{\circ} \mathrm{N}-$ $\left.135.02^{\circ} \mathrm{E}, M=7.2 ; 4\right) 19$ October $1996,31.89^{\circ} \mathrm{N}-131.47^{\circ} \mathrm{E}$, $M=7.0$. The epicenters of these earthquakes are shown as stars in Fig. 6.

\section{Discussion}

An attempt has been made in this paper to check the effect of zonation on the applicability of the regional time- and magnitude predictable seismicity model. In previous studies (Papazachos et al., 1997a, b) it had been observed that, when the model was applied in areas with complex seismicity patterns and tectonics (e.g. Philippines, Taiwan, New Hebrides), the accurate definition of seismogenic regions was important, but it was not very critical on the main features and the results of this model.

There are, however, some uncertainties in the application of this model that concern the long-term clustering of earthquakes and the interaction between adjacent faults (see Papazachos et al., 1997a, for detailed discussion).

Long-term clustering has been identified by Ambraseys (1989) in some areas in Middle East (East Anatolian Fault, Zagros). He concluded that deformations associated with earthquakes in these zones seem to occur every few hundred years, during relatively short paroxysmal periods of strong events. It is, thus, quite difficult to estimate future activity on the basis of a data sample of past events, depending upon whether the sample of observations has been taken from a high or low period of current activity. On the other hand, it has been shown that the most recent past may be a better indicator of future seismic activity (McGuire and Barnhard, 1981). The above mentioned conclusions show that the results depend strongly on the data on which they are based. 
As regards the interaction between adjacent regions, it has to be said that it remains a major problem which attracts the attention of many scientists who use stochastic simulations as well as multivariate stress release models to quantify the stress transfer between regions (Suzuki and Kiremidjan, 1991).

In the present paper it has been shown that the basic feature of the model, that is, the positive dependence of the return period, $T$, on the magnitude, $M_{p}$, of the preceding mainshock, exists independently of the shape or the surface of the region considered, since it has been found that in the majority of the cases in both areas studied, the $c$-values were positive. This result enables a tentative seismic hazard assessment in areas where no zonation has ever been attempted. More accurate seismic hazard studies require a detailed zonation based on as much data as possible.

Acknowledgments. The author thanks Prof. B. Papazachos for his valuable comments and discussion during all stages of this study, as well as the two reviewers who helped to improve the text and clarify some vague points. Thanks are also due to Ch. Papaioannou, C. Papazachos and E. Scordilis who provided the programs necessary to process the data. This work has been partially supported by the Project 10530/6.10.97 of the General Secretariat of Research and Technology of Greece.

\section{References}

Abe, K., Magnitudes of large earthquakes from 1904 to 1980, Phys. Earth Planet. Inter., 27, 72-93, 1981.

Ambraseys, N. N., Temporal seismic quiescence: SE Turkey, Geophys. J., 96, 311-331, 1989.

Ando, M., Source mechanisms and tectonic significance of historical earthquakes along the Nankai Trough, Japan, Tectonophys., 27, 119140, 1975.

Kagan, Y. Y. and D. D. Jackson, Seismic gap hypothesis: Ten years after, J. Geophys. Res., 96, 21419-21431, 1991.

Karakaisis, G. F., M. C. Kourouzidis, and B. C. Papazachos, Behaviour of seismic activity during a single seismic cycle, in Proc. Intern. Conf. Earthquake Prediction: State-of-the-Art, Strasbourg, 15-18 October 1991, France, pp. 47-54, 1991.

McGuire, R. K. and J. Barnhard, Effects of temporal variations in seismicity on seismic hazards, Bull. Seism. Soc. Am., 71, 321-334, 1981.

Mogi, K., Earthquake Prediction, 355 pp., Academic Press, Tokyo, 1985.

Molnar, P., Earthquake recurrence intervals and plate tectonics, Bull. Seism. Soc. Am., 69, 115-133, 1979.
Pacheco, J. F. and L. R. Sykes, Seismic moment catalog of large shallow earthquakes, 1900 to 1989, Bull. Seism. Soc. Am., 82, 1306-1349, 1992.

Papazachos, B. C., A time-predictable model for earthquakes in Greece, Bull. Seism. Soc. Am., 79, 77-84, 1989.

Papazachos, B. C. and Ch. A. Papaioannou, Long-term earthquake prediction in the Aegean area based on a time and magnitude predictable model, Pure Appl. Geophys., 140, 593-612, 1993.

Papazachos, B. C. and Ch. A. Papaioannou, Seismic hazard in Greece based on new seismotectonic data, in Abstracts IASPEI 29 th General Assembly, Thessaloniki, 18-28 August 1997, p. 294, 1997.

Papazachos, B. C. and C. B. Papazachou, The Earthquakes of Greece, 304 pp., Ziti Publications, Thessaloniki, 1997.

Papazachos, B. C., E. E. Papadimitriou, G. F. Karakaisis, and T. M. Tsapanos, An application of the time- and magnitude-predictable model for the long-term earthquake prediction of strong shallow earthquakes in the Japan area, Bull. Seism. Soc. Am., 84, 426-437, 1994.

Papazachos, B. C., E. E. Papadimitriou, G. F. Karakaisis, and D. G. Panagiotopoulos, Long-term earthquake prediction in the circumPacific convergent belt, Pure Appl. Geophys., 149, 173-217, 1997a.

Papazachos, B. C., G. F. Karakaisis, E. E. Papadimitriou, and Ch. A. Papaioannou, The regional time and magnitude predictable model and its application to the Alpine-Himalayan belt, Tectonophys., 271, 295-323,. 1997b.

Papazachos, B. C., G. F. Karakaisis, and P. M. Hatzidimitriou, Time dependent seismicity in Greece, in Abstracts IASPEI 29th General Assembly, Thessaloniki, 18-28 August 1997, p. 78, 1997c.

Papazachos, C. B. and E. E. Papadimitriou, Evaluation of the global applicability of the time and magnitude predictable seismicity model, Bull. Seism. Soc. Am., 87, 799-808, 1997.

Rikitake, T., Recurrence of great earthquakes at subduction zones, Tectonophys., 35, 335-362, 1976.

Rikitake, T., Current Research in Earthquake Prediction I, 383 pp., Reidel Publ. Co., Dordrecht, The Netherlands, 1982.

Shimazaki, K. and T. Nakata, Time-predictable recurrence model for large earthquakes, Geophys. Res. Lett., 7, 279-282, 1980.

Suzuki, S. and A. S. Kiremidjan, A random slip rate model for earthquake occurrences with Bayesian parameters, Bull. Seism. Soc. Am., 81, 781-795, 1991.

Tsapanos, T. M., E. M. Scordilis, and B. C. Papazachos, A Global Catalogue of Strong Earthquakes, 90 pp., Publ. Geophys. Lab., Univ. of Thessaloniki, 1990.

Utsu, T., A catalogue of large earthquakes $(M>6)$ and damaging earthquakes in Japan for the years 1885-1925, in Historical Seismograms and Earthquakes of the World, edited by W. H. K. Lee, H. Meyers, and K. Shimazaki, pp. 150-161, Academic Press, London, 1988.

G. F. Karakaisis (e-mail: karakais@geo.auth.gr) 